OPEN ACCESS

Edited by:

Tao Xiao,

Shenzhen University, China

Reviewed by:

Vicente Javier Clemente-Suárez, European University of Madrid, Spain

Gabriele Arnulfo,

University of Genoa, Italy

*Correspondence:

Qing Gao

gaoqing@uestc.edu.cn

tThese authors have contributed equally to this work

Specialty section:

This article was submitted to Movement Science and Sport

Psychology,

a section of the journal

Frontiers in Psychology

Received: 28 January 2020

Accepted: 25 August 2020 Published: 25 September 2020

Citation:

Yang C, Luo N, Liang M, Zhou S, Yu Q, Zhang J, Zhang M, Guo J, Wang $H, Y u J$, Cui Q, Chen H and Gao Q (2020) Altered Brain Functional Connectivity Density in Fast-Ball Sports Athletes With Early Stage of Motor Training.

Front. Psychol. 11:530122. doi: 10.3389/fpsyg.2020.530122

\section{Altered Brain Functional Connectivity Density in Fast-Ball Sports Athletes With Early Stage of Motor Training}

\author{
Chengbo Yang ${ }^{1 t}$, Ning Luo ${ }^{2 t}$, Minfeng Liang ${ }^{2}$, Sihong Zhou ${ }^{2}$, Qian $\mathrm{Yu}^{3}$, Jiabao Zhang ${ }^{2}$, \\ Mu Zhang', Jingpu Guo', Hu Wang' ${ }^{1}$, Jiali Yu' ${ }^{2}$, Qian Cui ${ }^{5}$, Huafu Chen ${ }^{6,7}$ and Qing Gao ${ }^{2 *}$ \\ ${ }^{1}$ The Third Department of Physical Education and Training, Chengdu Sport University, Chengdu, China, ${ }^{2}$ School \\ of Mathematical Sciences, University of Electronic Science and Technology of China, Chengdu, China, ${ }^{3}$ Exercise and Mental \\ Health Laboratory, School of Psychology, Shenzhen University, Shenzhen, China, ${ }^{4}$ Information Technology Center, Chengdu \\ Sport University, Chengdu, China, ${ }^{5}$ School of Public Affairs and Administration, University of Electronic Science \\ and Technology of China, Chengdu, China, ${ }^{6}$ Ministry of Education Key Lab for Neuroinformation, High-Field Magnetic \\ Resonance Brain Imaging Key Laboratory of Sichuan Province, University of Electronic Science and Technology of China, \\ Chengdu, China, ${ }^{7}$ The Clinical Hospital of Chengdu Brain Science Institute, School of Life Sciences and Technology, \\ University of Electronic Science and Technology of China, Chengdu, China
}

The human brain shows neuroplastic adaptations caused by motor skill training. Of note, there is little known about the plastic architecture of the whole-brain network in resting state. The purpose of the present study was to detect how motor training affected the density distribution of whole-brain resting-state functional connectivity (FC). Resting-state functional magnetic resonance imaging data was assessed based on a comparison of fast-ball student athletes (SA) and non-athlete healthy controls (NC). The voxel-wise data-driven graph theory approach, global functional connectivity density (gFCD) mapping, was applied. Results showed that the SA group exhibited significantly decreased gFCD in brain regions centered at the left triangular part of the inferior frontal gyrus (IFG), extending to the opercular part of the left IFG and middle frontal gyrus compared to the NC group. In addition, findings suggested the idea of an increased neural efficiency of athletes' brain regions associated with attentional-motor modulation and executive control. Furthermore, behavioral results showed that in the SA group, faster executive control reaction time relates to smaller gFCD values in the left IFG. These findings suggested that the motor training would decrease the numbers of FC in IFG to accelerate the executive control with high attentional demands and enable SA to rapidly focus the attention to detect the intriguing target.

Keywords: athlete training, global functional connectivity density, resting-state functional magnetic resonance imaging, attention, neuroplasticity

\section{INTRODUCTION}

The brain of skilled elite athletes who have improved sport performance reveals neuroplastic adaptations caused by motor skill training (Gao et al., 2019; Chen et al., 2020; Jung et al., 2020). It has attracted special attention from researchers exploring how and where the structural and functional plasticity of the brain occurs in the course of motor skill training using imaging 
techniques [e.g., functional magnetic resonance imaging (fMRI) and functional near infrared spectroscopy (fNIRs)] (Crawford et al., 2019; Yu et al., 2020; Yue et al., 2020a,b). In particular, fMRI is widely accepted as an effective method to help researchers to understand the physiological mechanisms of brain plasticity via motor training; furthermore, findings might be practically meaningful for improving athletic performance (Gao et al., 2019; Zhang et al., 2019). However, it is largely unknown how brain functions change in the process of motor training (Lappi, 2015). Some researchers attempted to investigate the difference in brain activity between athletes and non-athlete controls (NC) during certain tasks (Gao et al., 2019), with different regions of task-induced activation between the two groups that are being reported. For instance, in the visual smooth pursuit task, football players showed greater activation in the oculomotor region of the cerebellar vermis and areas of the frontal eye fields than NC, suggesting visual motor skill is required for elite football players to successfully execute complex movement during games (Kellar et al., 2018). In addition, enhanced inferior parietal/frontal gyrus activity has been proposed in basketball players anticipating a free-throw task (Wu et al., 2013). Previous research suggested that when familiar sports environmental sounds surrounded, elite athletes tend to have greater activation in somatosensory regions but less activation in brain regions, which associated with perception and motor planning and processing (Woods et al., 2014). Except for the aforementioned results, a decreased brain activation in athletes (e.g., archery and table tennis) was reported across various cognitive tasks (e.g., mental rehearsal and visualspatial task) compared to non-experts (Chang et al., 2011; Guo et al., 2017).

Resting-state fMRI (rsfMRI) is the commonly used method in the human brain map to evaluate intrinsic spontaneous fluctuations in the blood oxygen level-dependent (BOLD) signal in the resting or task-free state (Gao et al., 2013). This innovative and effective method was widely used in previous studies to investigate intrinsic brain plasticity or regional interaction induced by motor training. A study which implemented seedbased functional connectivity (FC) in distance runners shows increased connectivity between the fronto-parietal network and brain regions for execution functions compared to non-expert runners (Raichlen et al., 2016). These functional alterations in fronto-parietal connectivity were also found in badminton athletes (Di et al., 2012; Xu et al., 2016). In addition, similar results are found in elite karate players, suggesting brain areas associated with movement planning and visual perception having increased connectivity (Duru and Balcioglu, 2018). Our recent study using seed-based stepwise FC showed that fast-ball athletes were reported with a significantly smaller optimal connectivity distance from seed regions to the dorsal attention network (DAN) and larger optimal connectivity distance to the default mode network (DMN; Gao et al., 2019). Given the evidences above, changes in resting-state-FC brain plasticity occur in different brain areas across various sport events.

However, seed-based methods involved with the a priori selection of appropriate seed regions are unable to fully characterize the brain functional connectome as well as get a whole picture on the plastic architecture of the whole-brain network (Joel et al., 2011). Recently, functional connectivity density (FCD) mapping, a voxel-wise data-driven graph theory approach, has been established to determine the density distribution of whole-brain resting-state FC (Tomasi and Volkow, 2011; Huang et al., 2018). It measures the number of functional connections of a given voxel with the remaining voxels in the whole brain. The brain regions with high FCD values are considered as functional hubs of the human brain, which play a very important role in brain function (Tomasi and Volkow, 2011). Thus, this approach is a reliable and effective method and has been increasingly accepted in research detecting biomarkers of neuropsychiatric disorders through resting-state functional network alterations, including schizophrenia (Huang et al., 2018), major depressive disorder (Zhang et al., 2016; Zou et al., 2016), migraine (Gao et al., 2016), and aging brain (Li et al., 2019).

Here, we aimed to explore the neuroplasticity on brain functional organization induced by motor training using the global FCD (gFCD) approach. Fast-ball student athletes (SA) who play badminton, tennis, and table tennis were recruited. In fastball sports like badminton, tennis, and table tennis, the players require well-refined hand-eye coordination and visuospatial ability to achieve improved sports-related cognitions such as perception, focus, anticipation, planning, and fast responses (Di et al., 2012; Wolf et al., 2014). We started with an assumption that the fast-ball athletes would show changes in FC architecture in brain regions related to the visual attention and visual-motor coordination. To test whether the brain gFCD values relate to attentional processes, all subjects were asked to perform a revised attention network test (ANT) processing. The relationships between $\mathrm{gFCD}$ and reaction time of each attention subnetwork of alerting, orienting, and executive control were also investigated.

\section{MATERIALS AND METHODS}

\section{Participants}

Forty-two SA majoring in fast-ball sports (badminton, tennis, and table tennis) were recruited from Chengdu Sport University. All SA were required to pass the college entrance examination for sports majors in Sichuan province of China before entering Chengdu Sport University. The examination consisted of 40 points of the sport-specific test and 20 points of three physical fitness events, including 100-m run, standing triple jump, and standing shot put. The full mark of all test items is based on or slightly higher than the Standard of Technical Grade of Athletes issued by the General Administration of Sport of China. For example, the full mark of $100 \mathrm{~m}$ is $11.54 \mathrm{~s}$, and the total score of all four test items has to exceed 85 points. After entering Chengdu Sport University, SA have been engaged in the fast ball-specific training. The training duration was less than 3 years, and the training frequency was less than $25 \mathrm{~h}$ per week. Thirty-nine NC with matched age, gender, and education level were also recruited. Except the sports-required course in the university, the NC group spent no more time on physical activity. All participants were tested handedness using the Chinese version of the EdinburghHandedness Questionnaire (coefficients > 50) (Oldfield, 1971), and all showed right-handedness. Participants had no history of 
neurological or psychiatric diseases or concussions. The study protocol was approved by the research ethical committee of School of Life Sciences and Technology, University of Electronic Science and Technology of China. Participants were provided written informed consent prior to any assessment.

\section{Data Acquisition}

Magnetic resonance imaging (MRI) images were acquired on a 3.0 T GE Signa MR750 system (GE Healthcare, Milwaukee) with an 8-channel phased array head coil. High-resolution 3D T1weighted anatomical images were obtained in axial orientation using a 3D spoiled gradient-recalled (SPGR) sequence. The acquisition parameters were as follows: $\mathrm{TR}=5.97 \mathrm{~ms}$, $\mathrm{TE}=1.96 \mathrm{~ms}$, field of view $(\mathrm{FOV})=240 \mathrm{~mm} \times 240 \mathrm{~mm}$, flip angle $=12^{\circ}$, matrix size $=512 \times 512,156$ slices, and voxel size $=1 \mathrm{~mm} \times 1 \mathrm{~mm} \times 1 \mathrm{~mm}$. Resting-state fMRI images were acquired using a gradient-recalled echo planar imaging (EPI) sequence. The parameters were $\mathrm{TR}=2000 \mathrm{~ms}$, $\mathrm{TE}=30 \mathrm{~ms}, \mathrm{FOV}=220 \mathrm{~mm} \times 220 \mathrm{~mm}$, flip angle $=90^{\circ}$, matrix size $=64 \times 64,43$ transverse slices without a slice gap, voxel size $=3.75 \mathrm{~mm} \times 3.75 \mathrm{~mm} \times 3.2 \mathrm{~mm}$, and a total of 266 volumes for each subject. During the scan, the subjects were instructed to lie down with their eyes closed, not to think of anything in particular, and not to fall asleep. Padded foams were used to restrict head motion, and earplugs were used to attenuate scanner noise.

\section{Data Preprocessing}

Conventional fMRI data preprocessing was performed using Data Processing Assistant for Resting-State fMRI software (DPARSF, Advanced Edition, V4.5). ${ }^{1}$ The first 10 volumes of each subject were discarded to ensure steady-state longitudinal magnetization. The remaining 256 resting-state fMRI images were first corrected for the acquisition time delay between different slices and then realigned to the first volume to correct for head motion. We required that the transient movement during the scanning was no more than $1.0 \mathrm{~mm}$ of translation and $1.0^{\circ}$ of rotation. The images were further spatially normalized into a standard stereotaxic space at $3 \mathrm{~mm} \times 3 \mathrm{~mm} \times 3 \mathrm{~mm}$, using the EPI template in the Statistical Parametric Mapping software (SPM8). The images were not smoothed to avoid introducing artificial local spatial correlations. Images were then linearly detrended and were corrected using linear regression to remove the possible spurious variances including 24 head-motion parameters, averaged signals from cerebrospinal fluid, and white matter. The residuals of these regressions were temporally bandpass filtered $(0.01<f<0.08 \mathrm{~Hz})$ to reduce low-frequency drifts and physiological high-frequency respiratory and cardiac noises. Finally, scrubbing with the interpolation method was used to remove the bad points of the data. Since FC analysis is sensitive to gross head motion effects (Power et al., 2012), the mean frame-wise displacement (FD) was calculated to further determine the comparability of head movement across groups. The largest FD obtained from the subjects was less than $0.2 \mathrm{~mm}$ (which was $0.178 \mathrm{~mm}$ ).

\footnotetext{
${ }^{1}$ http://www.restfmri.net/forum/
}

\section{Global Functional Connectivity Density Analysis}

Global functional connectivity density is defined as the number of statistically significant FCs between a given voxel and the rest of voxels across the whole brain in a binary network (Tomasi and Volkow, 2010). It is capable of quantifying the importance/centrality of a given voxel within the whole-brain network (Tomasi and Volkow, 2010). The voxel-wise gFCD maps were computed by an in-house program coded in MATLAB (The MathWorks, Natick, MA) according to the approach introduced by Tomasi and Volkow (2010). Briefly, we calculated the Pearson's correlation $(r)$ between brain voxels that limited in a gray matter mask based on the automated anatomical labeling (AAL) atlas to obtain a whole-brain FC map at the voxel level. Two voxels were considered to be connected if their Pearson's correlation coefficient of the two voxels was greater than a given correlation threshold $T c=0.6$ according to the significant level of $p<0.01$ (Bonferroni multiple correction), in order to eliminate the weak correlation which may be caused by noise (Tomasi and Volkow, 2010; Huang et al., 2018). Here, the significant level was set for testing the hypothesis of no functional connection against the alternative that there is a functional connection (nonzero correlation). The value of gFCD was defined of the global number of functional connections $n_{i}$, between voxel $i$ and all other voxels in the brain. For each participant, gFCD maps were further standardized by the total number of edges in the whole brain (Li et al., 2018) and were spatially smoothed with full-width at half-maximum (FWHM) $=8 \mathrm{~mm}$ to minimize the individual overall differences of gFCD values (Tomasi and Volkow, 2011).

\section{The Attention Network Test}

To test whether the altered FC network architecture relates to attentional processes, all subjects were asked to perform a revised ANT (Wang et al., 2016), as shown in Figure 1. Briefly, the stimuli were presented via E-Prime 2.0 (Psychology Software Tools,

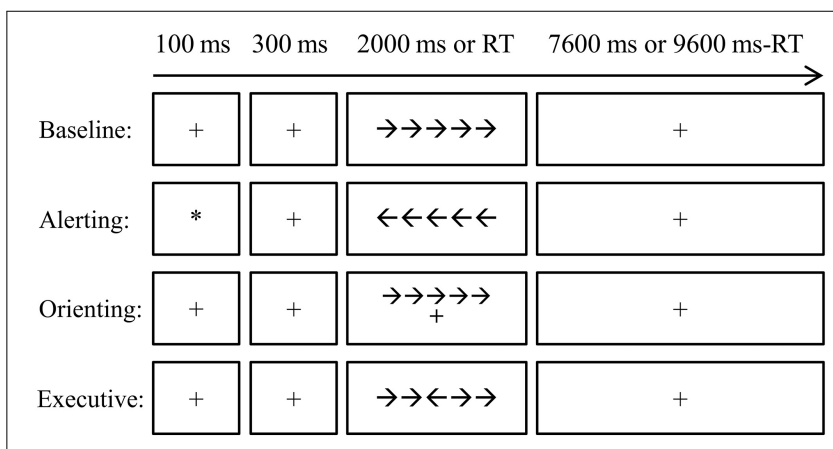

FIGURE 1 | The diagram for the attention network test (ANT) procedure. The four conditions, i.e., baseline, alerting, orienting, and executive control, were arranged in separated blocks. Each trial started with a cue (the alerting condition) or a fixation with $100 \mathrm{~ms}$. After a 300-ms interval, the target was presented for 2,000 ms or disappeared after one key was pressed. A fixation was then presented until the end of that trial. Each trial lasted for $10 \mathrm{~s}$. RT, reaction time. 
Inc $^{2}$ ) on a Lenovo PC. Responses were collected via Q (for left targets) and $\mathrm{P}$ (for right targets) on the keyboard. All participants completed four blocks (alerting block, orienting block, executive control block, and baseline block) of the ANT. The order of the four blocks was counterbalanced across subjects. Each block took $424 \mathrm{~s}$, which contained a buffer time of $4 \mathrm{~s}$, two practice trials of $10 \mathrm{~s}$, and 40 experimental trials of $10 \mathrm{~s}$. As shown in Figure 1, each trial began with a fixation or cue for $100 \mathrm{~ms}$ which was followed by a 300-ms fixation. After that, a target (congruent or incongruent, central, or spatial) appeared for $2000 \mathrm{~ms}$ or until the participant pressed a key. Lastly, another fixation was presented to ensure that the overall time of one trial was $10,000 \mathrm{~ms}(0.1 \mathrm{~Hz})$. The subjects were asked to judge the direction of the third arrow (the central one) by pressing $Q$ if it points to the left and $\mathrm{P}$ if it points to the right. The attention network scores (ANSs) were computed to measure the reaction time of each attention subnetwork of alerting, orienting, and executive control compared to the baseline (Wang et al., 2016).

\section{Statistical Analysis}

We used two-sample $t$-test analysis to compare the group differences of gFCD between the two groups. Age, gender, and education were considered as covariate variables. The statistically significant threshold of the gFCD was set for multiple comparisons at the cluster level with $p<0.05$ (AlphaSim corrected). This correction was conducted using the DPARSF software.

To investigate whether the altered gFCD was associated with the behavioral reaction time of each attention subnetwork of alerting, orienting, and executive control, relationships between gFCD values in regions showing significant group differences and the reaction time of the three attention subnetworks were further detected by partial correlation analysis. The partial correlation analysis was performed for the SA group and the NC group, separately, with age and gender as confounding factors.

\section{RESULTS}

\section{Demographic Data}

The demographic data of the recruited subjects are shown in Table 1. The athlete group and the control group did not differ significantly in age (Mann-Whitney U-test, $p=0.17$ ), gender (Pearson $\chi^{2}$ test, $p=0.35$ ), education (Mann-Whitney U-test, $p=0.58$ ), or mean FD (Mann-Whitney U-test, $p=0.94$ ).

\section{gFCD in Athletes and Controls}

Figure 2 shows the spatial distribution maps of the average gFCD in the SA group and the NC group, respectively. In both the SA and NC groups, the prefrontal cortex, posterior cingulate cortex, precuneus, and occipital cortex had relatively high gFCD values, as reported in previous studies (Tomasi and Volkow, 2010; Luo et al., 2014; Huang et al., 2018). Figure 3 (left) shows the significantly different gFCD between the groups $(p<0.05$, AlphaSim corrected). The SA group exhibited

${ }^{2}$ http://www.pstnet.com significantly decreased gFCD in brain regions centered at the left triangular part of the inferior frontal gyrus (IFG), extending to the left opercular part of the IFG and middle frontal gyrus (MFG) compared to the NC group. Table 2 presented the Montreal Neurological Institute (MNI) coordinates of peak voxels and statistical $t$-values of the brain regions with significantly different gFCD between the two groups. The positive $t$-values represented that the NC group had higher gFCD values than the SA group. No significantly increased gFCD in the SA group compared with the NC group was found.

\section{ANT Scores and Relationships With gFCD}

Figure 3 (right) shows the significant correlations between the gFCD values and the reaction time of executive control in the SA group. In the SA group, the gFCD values in the left triangular part of IFG showed significant positive correlations with the executive control reaction time. In the NC group, subjects had relatively low values in reaction time, and the within-group dispersion of the reaction time was relatively small. The significant correlation between the gFCD values and the reaction time was not detected in the NC group.

\section{DISCUSSION}

The frontal cortex is one of the most crucial areas related to highlevel functional integration (Woods et al., 2014). The inferior and middle frontal cortex especially contributed to the visual attention modulation and executive functions (Woods et al., 2014; Guo et al., 2017). In the task research in athletes, expert athletes had less activation in IFG and MFG when listening to familiar sports environmental sounds, suggesting the neural efficiency in these brain regions associated with perception and motor planning and processing (Woods et al., 2014). During the motor reaction and the visuo-spatial tasks, formula racing-car drivers recruited distributed networks including the middle and inferior frontal cortices, which devoted to executive functions (Bernardi et al., 2013). However, professional drivers recruited these regions to a significantly smaller extent as compared to naïve subjects (Bernardi et al., 2013). As support, table tennis athletes perform the visuo-spatial task with less brain activation in bilateral MFG than non-athletes (Guo et al., 2017). A similar finding was also observed in expert hockey players, suggesting neural efficiency in these brain regions for action decision as they were presented videos for shooting a puck toward a hockey goal (Olsson and Lundstrom, 2013). However, there were controversial results in some tasks. For instance, an increased activity in the inferior parietal lobule and IFG was detected in basketball athletes performing a freethrow task (Wu et al., 2013). Therefore, the task-related results demonstrated IFG and MFG as functional hubs of visual motor integration, motor control, and executive functions. In addition, the results suggested their functional plasticity induced by motor skill training.

The rsfMRI results are independent of task. The task-free approach can explore the intrinsic neural plasticity induced by 
TABLE 1 | Demographics of the subjects.

\begin{tabular}{|c|c|c|c|c|c|c|}
\hline & Age (years) & Gender (female/male) & Education (years) & Training time (h/week) & Duration (years) & Mean FD (mm) \\
\hline $\mathrm{SA}(n=42)$ & $20.43 \pm 0.59$ & $12 / 30$ & $14.57 \pm 0.67$ & $17.17 \pm 0.83$ & $1.95 \pm 0.08$ & $0.08 \pm 0.02$ \\
\hline $\mathrm{NC}(n=39)$ & $20.33 \pm 1.33$ & $15 / 24$ & $15.05 \pm 1.43$ & - & - & $0.08 \pm 0.03$ \\
\hline$P$ value & $0.1736^{a}$ & $0.3454^{b}$ & $0.5803^{a}$ & - & - & $0.9363^{a}$ \\
\hline
\end{tabular}

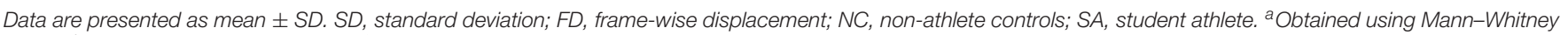
$U$-test. ${ }^{b}$ Obtained using the Pearson $\chi^{2}$ test.

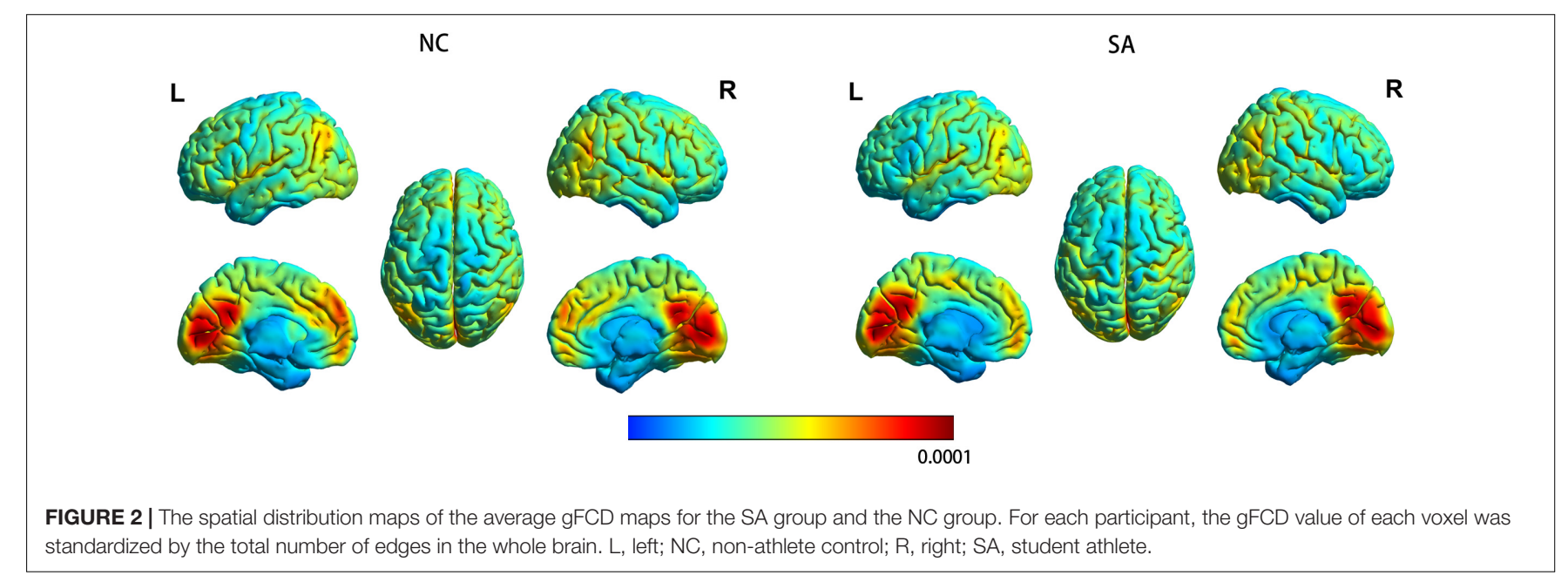

FIGURE 2 | The spatial distribution maps of the average gFCD maps for the SA group and the NC group. For each participant, the gFCD value of each voxel was standardized by the total number of edges in the whole brain. L, left; NC, non-athlete control; R, right; SA, student athlete.

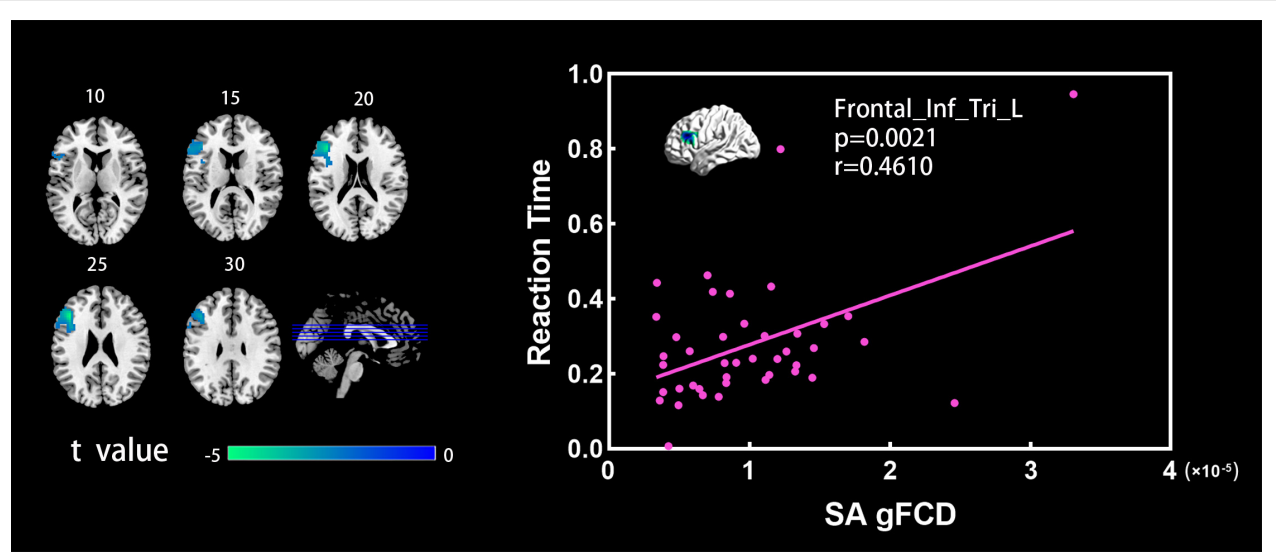

FIGURE 3 | (Left) Brain regions showing significant differences between the SA and NC groups in the gFCD analysis (two sample $t$-test, $p<0.05$, GRF corrected). Numbers above each image refer to the $z$-plane coordinates of the MNI space. (Right) Significant correlation between reaction time and gFCD values in the SA group. Frontal_Inf_Tri_L, left triangular part of inferior frontal gyrus.

TABLE 2 | Brain regions showed significantly different gFCD between the athlete group and the control group.

\begin{tabular}{|c|c|c|c|c|}
\hline Brain regions & Hem & Peak coordinates & Cluster size & $T$-value \\
\hline Inferior frontal gyrus, triangular & $\mathrm{L}$ & $(-39,27,24)$ & 328 & 5.63 \\
\hline Inferior frontal gyrus, opercular & $L$ & $(-57,27,18)$ & 68 & 3.83 \\
\hline Middle frontal gyrus & $\mathrm{L}$ & $(-54,36,21)$ & 144 & 3.72 \\
\hline
\end{tabular}

motor skill training. There has been local seed-based FC analysis, which showed altered FC between the athlete group and controls within the left frontoparietal network (Di et al., 2012). In the athlete group, an enhanced FC between the left superior parietal lobule and left MFG (BA6) was detected, whereas a lower FC between the left superior parietal lobule and the left MFG (BA9) was observed compared to the control group (Di et al., 2012). In addition, previous research was found that distance runners 
exhibited enhanced connectivity between the right parietal cortex and MFG (Raichlen et al., 2016).

Global functional connectivity density is a powerful framework to characterize the FC architecture within the whole-brain network without any prior hypothesis (Tomasi and Volkow, 2011). From the point of view of the whole-brain resting-state FC, our results showed decreased gFCD values in the left IFG and MFG in the fast-ball SA group compared to the NC group. This is a distinct but complementary information on the brain plasticity caused by motor skill training in fast-ball SA. Such results demonstrated reduced connections between these brain regions and the remaining brain voxels in the SA group. The findings were consistent with the previous research, regardless of skilled groups, such as racing-car drivers (Bernardi et al., 2013), table tennis players (Guo et al., 2017), basketball players (Pi et al., 2019), golfers (Milton et al., 2007), and musicians (Haslinger et al., 2004; Meister et al., 2005). The possible explanation could be that athletes' brain might exhibit an improved neural efficiency in the brain regions associated with attentional-motor modulation and executive control along with a reduced "resource consumption" (Rypma and Prabhakaran, 2009; Bernardi et al., 2013; Guo et al., 2017; Sommer et al., 2018; Zhang et al., 2019).

Importantly, we further detected a positive correlation between gFCD values in the left triangular part of IFG and reaction time of the executive control in the SA group. Of the three attention subnetworks, the executive control network is very useful for producing top-down regulation and thus is related to executive control (Petersen and Posner, 2012). This system was presented under the heading of target detection (Petersen and Posner, 2012). It is related to the limited capacity of the attention system, and to awareness itself, and has often been called focal attention (Petersen and Posner, 2012). Fast-ball sport events require athletes to execute actions in a short time (Ermutlu et al., 2015), in terms of wellrefined hand-eye coordination and visuospatial ability to achieve enhanced perception, concentration, anticipation, planning, and fast response requirements (Di et al., 2012; Gao et al., 2019). Our results suggested that faster executive control reaction time was associated with smaller gFCD values in the left triangular part of IFG. The behavioral results further supported that in the SA group, shorter reaction time of executive control was associated with smaller gFCD values, which referred to the numbers of FC between a given voxel and the rest of voxels across the whole brain. The findings implied that motor skill training results in a decrease in the number of FC in the left triangular part of IFG, which athletes are interested in developing reaction capacity, movement planning, and execution with high attentional demands, and focus the attention to the target detection (Wolf et al., 2014). Of note, sample size was relatively low in the present study, and only the student-athletes who were trained in fast ball of badminton, tennis, and table tennis were recruited. Our findings only limited to the fast-ball sports athletes with early stage of motor training.

\section{CONCLUSION}

Using resting-state functional imaging techniques and graph theory approach, the present study revealed that compared with the NC group, the SA group exhibited significantly decreased gFCD in IFG and MFG. As supportive of the behavioral data, faster executive control reaction time was associated with smaller gFCD values in the left triangular part of IFG in the SA group. The findings supported the idea of an increased neural efficiency of athletes' brain in the brain regions associated with attentional-motor modulation and executive control and implied the training-induced neuroplasticity occurring in these brain regions. In addition, our findings were based on the restingstate brain functional network, which was independent of specific tasks. The neural mechanisms of functional adaptations in the athletes' brain that made their exceptional performance possible might have potential applications in designing optimal sports coaching methods, in overcoming learning disabilities, and in neurological rehabilitation (Lappi, 2015).

\section{DATA AVAILABILITY STATEMENT}

The datasets generated for this study are available on request to the corresponding author.

\section{ETHICS STATEMENT}

The study protocol was approved by the research ethical committee of School of Life Sciences and Technology, University of Electronic Science and Technology of China. The patients/participants provided their written informed consent to participate in this study. Written informed consent was obtained from the individual(s) for the publication of any potentially identifiable images or data included in this article.

\section{AUTHOR CONTRIBUTIONS}

CY, NL, and QG contributed to the conception and design of the research. MZ, JG, HW, and ML collected the data. ML, SZ, and JZ applied the statistical analysis. NL and CY analyzed and interpreted the data. CY, NL, and QG wrote the manuscript. QG and $\mathrm{HC}$ edited the manuscript. QG was responsible for the overall project. QY edited and modified the manuscript. All authors contributed to the article and approved the submitted version.

\section{FUNDING}

The work was supported by the National Natural Science Foundation of China $(61573085,61533006,61572112,81771919$, and U1808204), the Science and Technology Support Program of Sichuan Province (2018JY0562 and 2019YJ0185), and China Scholarship Council (201506075025). 


\section{REFERENCES}

Bernardi, G., Ricciardi, E., Sani, L., Gaglianese, A., Papasogli, A., Ceccarelli, R., et al. (2013). How skill expertise shapes the brain functional architecture: an fMRI study of visuo-spatial and motor processing in professional racing-car and naive drivers. PLoS One 8:e77764. doi: 10.1371/journal.pone.0077764

Chang, Y., Lee, J. J., Seo, J. H., Song, H. J., Kim, Y. T., Lee, H. J., et al. (2011). Neural correlates of motor imagery for elite archers. NMR Biomed. 24, 366-372.

Chen, L. Z., Yuan, X., Zhang, Y., Zhang, S., Zou, L., Yang, L., et al. (2020). Brain functional specialization is enhanced among tai chi chuan practitioners. Arch. Phys. Med. Rehabil. 101, 1176-1182.

Crawford, L., Zou, L., and Loprinzi, P. D. (2019). Oxygenation of the prefrontal cortex during memory interference. J. Clin. Med. 8:2055. doi: 10.3390/ jcm8122055

Di, X., Zhu, S., Jin, H., Wang, P., Ye, Z., Zhou, K., et al. (2012). Altered resting brain function and structure in professional badminton players. Brain Connect. 2, 225-233.

Duru, A. D., and Balcioglu, T. H. (2018). Functional and structural plasticity of brain in elite karate athletes. J. Healthc. Eng. 2018:8310975. doi: 10.1155/2018/ 8310975

Ermutlu, N., Yücesir, I., Eskikurt, G., Temel, T., and Ý??oð̋lu-Alkaç, Ü (2015). Brain electrical activities of dancers and fast ball sports athletes are different. Cogn. Neurodyn. 9, 257-263. doi: 10.1007/s11571-014-9320-2

Gao, Q., Xu, F., Jiang, C., Chen, Z., Chen, H., Liao, H., et al. (2016). Decreased functional connectivity density in pain-related brain regions of female migraine patients without aura. Brain Res. 1632, 73-81. doi: 10.1016/j.brainres.2015.12. 007

Gao, Q., Xu, Q., Duan, X., Liao, W., Ding, J., Zhang, Z., et al. (2013). Extraversion and neuroticism relate to topological properties of resting-state brain networks. Front. Hum. Neurosci. 7:257. doi: 10.3389/fnhum.2013.00257

Gao, Q., Yu, Y., Su, X., Tao, Z., Zhang, M., Wang, Y., et al. (2019). Adaptation of brain functional stream architecture in athletes with fast demands of sensorimotor integration. Hum. Brain Mapp. 40, 420-431.

Guo, Z., Li, A., and Yu, L. (2017). "Neural efficiency" of athletes' brain during visuospatial task: an fMRI study on table tennis players. Front. Behav. Neurosci. 11:72. doi: 10.3389/fnbeh.2017.00072

Haslinger, B., Erhard, P., Altenmüller, E., Hennenlotter, A., Schwaiger, M., Gräfin von Einsiedel, H., et al. (2004). Reduced recruitment of motor association areas during bimanual coordination in concert pianists. Hum. Brain Mapp. 22, 206-215. doi: 10.1002/hbm.20028

Huang, H., Jiang, Y., Xia, M., Tang, Y., Zhang, T., Cui, H., et al. (2018). Increased resting-state global functional connectivity density of default mode network in schizophrenia subjects treated with electroconvulsive therapy. Schizophr. Res. 197, 192-199. doi: 10.1016/j.schres.2017.10.044

Joel, S. E., Caffo, B. S., van Zijl, P. C., and Pekar, J. J. (2011). On the relationship between seed-based and ICA-based measures of functional connectivity. Magn. Reson. Med. 66, 644-657. doi: 10.1002/mrm.22818

Jung, M., Zou, L., Yu, J. J., Ryu, S., Kong, Z., Yang, L., et al. (2020). Does exercise have a protective effect on cognitive function under hypoxia? A systematic review with meta-analysis. J. Sport Health Sci. [Epub ahead of print]. doi: 10.1016/j.jshs.2020.04.004

Kellar, D., Newman, S., Pestilli, F., Cheng, H., and Port, N. L. (2018). Comparing fMRI activation during smooth pursuit eye movements among contact sport athletes, non-contact sport athletes, and non-athletes. Neuroimage Clin. 18, 413-424. doi: 10.1016/j.nicl.2018.01.025

Lappi, O. (2015). The racer's brain - how domain expertise is reflected in the neural substrates of driving. Front. Hum. Neurosci. 9:635. doi: 10.3389/fnhum.2015. 00635

Li, H., Zhang, X., Sun, B., Jiang, S., Li, J., Liu, C., et al. (2019). BOLD-fMRI reveals the association between renal oxygenation and functional connectivity in the aging brain. Neuroimage 186, 510-517. doi: 10.1016/j.neuroimage.2018.11.030

Li, R., Wang, L., Chen, H., Guo, X., Liao, W., Tang, Y. L., et al. (2018). Abnormal dynamics of functional connectivity density in children with benign epilepsy with centrotemporal spikes. Brain Imaging Behav. 13, 985-994. doi: 10.1007/ s11682-018-9914-0

Luo, C., Tu, S., Peng, Y., Gao, S., Li, J., Dong, L., et al. (2014). Long-term effects of musical training and functional plasticity in salience system. Neural. Plast. 2014:180138.
Meister, I., Krings, T., Foltys, H., Boroojerdi, B., Müller, M., Töpper, R., et al. (2005). Effects of long-term practice and task complexity in musicians and nonmusicians performing simple and complex motor tasks: implications for cortical motor organization. Hum. Brain Mapp. 25, 345-352. doi: 10.1002/hbm. 20112

Milton, J., Solodkin, A., Hlustík, P., and Small, S. L. (2007). The mind of expert motor performance is cool and focused. Neuroimage 35, 804-813. doi: 10.1016/ j.neuroimage.2007.01.003

Oldfield, R. C. (1971). The assessment and analysis of handedness: the Edinburgh inventory. Neuropsychologia 9, 97-113. doi: 10.1016/0028-3932(71)90067-4

Olsson, C. J., and Lundstrom, P. (2013). Using action observation to study superior motor performance: a pilot fMRI study. Front. Hum. Neurosci. 7:819. doi: 10.3389/fnhum.2013.00819

Petersen, S. E., and Posner, M. I. (2012). The attention system of the human brain: 20 years after. Annu. Rev. Neurosci. 35, 73-89. doi: 10.1146/annurev-neuro062111-150525

Pi, Y. L., Wu, X.-H., Wang, F.-J., Liu, K., Wu, Y., Zhu, H., et al. (2019). Motor skill learning induces brain network plasticity: a diffusiontensor imaging study. PLoS One 14:e210015. doi: 10.1371/journal.pone.021 0015

Power, J. D., Barnes, K. A., Snyder, A. Z., Schlaggar, B. L., and Petersen, S. E. (2012). Spurious but systematic correlations in functional connectivity MRI networks arise from subject motion. Neuroimage 59, 2142-2154. doi: 10.1016/ j.neuroimage.2011.10.018

Raichlen, D. A., Bharadwaj, P. K., Fitzhugh, M. C., Haws, K. A., Torre, G. A., Trouard, T. P., et al. (2016). Differences in resting state functional connectivity between young adult endurance athletes and healthy controls. Front. Hum. Neurosci. 10:610. doi: 10.3389/fnhum.2016.00610

Rypma, B., and Prabhakaran, V. (2009). When less is more and when more is more: the mediating roles of capacity and speed in brain-behavior efficiency. Intelligence 37, 207-222. doi: 10.1016/j.intell.2008.12.004

Sommer, M., Häger, C. K., Boraxbekk, C. J., and Rönnqvist, L. (2018). Timing Training in female soccer players: effects on skilled movement performance and brain responses. Front. Hum. Neurosci. 12:311. doi: 10.3389/fnhum.2018. 00311

Tomasi, D., and Volkow, N. D. (2010). Functional connectivity density mapping. Proc. Natl. Acad. Sci. U.S.A. 107, 9885-9890. doi: 10.1073/pnas.1001414107

Tomasi, D., and Volkow, N. D. (2011). Association between functional connectivity hubs and brain networks. Cereb. Cortex 21, 2003-2013. doi: 10.1093/cercor/ bhq268

Wang, Y. F., Long, Z., Cui, Q., Liu, F., Jing, X. J., Chen, H., et al. (2016). Low frequency steady-state brain responses modulate large scale functional networks in a frequency-specific means. Hum. Brain Mapp. 37, 381-394. doi: $10.1002 / \mathrm{hbm} .23037$

Wolf, S., Brölz, E., Scholz, D., Ramos-Murguialday, A., Keune, P. M., Hautzinger, M., et al. (2014). Winning the game: brain processes in expert, young elite and amateur table tennis players. Front. Behav. Neurosci. 8:370. doi: 10.3389/fnbeh. 2014.00370

Woods, E. A., Hernandez, A. E., Wagner, V. E., and Beilock, S. L. (2014). Expert athletes activate somatosensory and motor planning regions of the brain when passively listening to familiar sports sounds. Brain Cogn. 87, 122-133. doi: 10.1016/j.bandc.2014.03.007

Wu, Y., Zeng, Y., Zhang, L., Wang, S., Wang, D., Tan, X., et al. (2013). The role of visual perception in action anticipation in basketball athletes. Neuroscience 237, 29-41. doi: 10.1016/j.neuroscience.2013.01.048

Xu, H., Wang, P., Ye, Z., Di, X., Xu, G., Mo, L., et al. (2016). The role of medial frontal cortex in action anticipation in professional badminton players. Front. Psychol. 7:1817. doi: 10.3389/fpsyg.2016.01817

Yu, Q., Herold, F., Becker, B., KluGah-Brown, B., Zhang, Y., Perrey, S., et al. (2020). Cognitive benefits of exercise interventions: an fMRI activation likelihood estimation meta-analysis. bioRxiv [Preprint]. doi: 10.1101/2020.07.04.187401

Yue, C., Zhang, Y., Jian, M., Herold, F., Yu, Q., Mueller, P., et al. (2020a). Differential effects of tai chi chuan (motor-cognitive training) and walking on brain networks: a resting-state fMRI study in chinese women aged 60 . Healthcare 8:67. doi: 10.3390/healthcare8010067

Yue, C., Zou, L., Mei, J., Moore, D., Herold, F., Müller, P., et al. (2020b). Tai chi training evokes significant changes in brain white matter network in older women. Healthcare 8:57. doi: 10.3390/healthcare8010057 
Zhang, B., Li, M., Qin, W., Demenescu, L. R., Metzger, C. D., Bogerts, B., et al. (2016). Altered functional connectivity density in major depressive disorder at rest. Eur. Arch. Psychiatry Clin. Neurosci. 266, 239-248. doi: 10.1007/s00406015-0614-0

Zhang, L., Qiu, F., Zhu, H., Xiang, M., and Zhou, L. (2019). Neural efficiency and acquired motor skills: an fMRI study of expert athletes. Front. Psychol. 10:2752. doi: 10.3389/fpsyg.2019.02752

Zou, K., Gao, Q., Long, Z., Xu, F., Sun, X., Chen, H., et al. (2016). Abnormal functional connectivity density in first-episode, drug-naive adult patients with major depressive disorder. J. Affect. Disord. 194, 153-158. doi: 10.1016/j.jad. 2015.12.081
Conflict of Interest: The authors declare that the research was conducted in the absence of any commercial or financial relationships that could be construed as a potential conflict of interest.

Copyright (c) 2020 Yang, Luo, Liang, Zhou, Yu, Zhang, Zhang, Guo, Wang, Yu, Cui, Chen and Gao. This is an open-access article distributed under the terms of the Creative Commons Attribution License (CC BY). The use, distribution or reproduction in other forums is permitted, provided the original author(s) and the copyright owner(s) are credited and that the original publication in this journal is cited, in accordance with accepted academic practice. No use, distribution or reproduction is permitted which does not comply with these terms. 